\title{
Dorieus und Kleomenes I. - Überlegungen zu Hdt. 5,39-48
}

\author{
Patrick Reinard
}

Dorieus von Sparta und seine gescheiterten Expeditionen nach Libyen und Sizilien haben aufgrund knapper Quellennachrichten in der Forschung nur wenig Beachtung gefunden. Der ausführlichste und, wie bereits Benediktus Niese anmerkte ${ }^{1}$, eigentlich einzige antike Bericht findet sich im fünften Buch der Historien Herodots. Selbiger kommt im siebten Buch (158 u. 205) nochmals kurz auf Dorieus zu sprechen und erwähnt im neunten Buch (10) dessen Sohn Euryanax. Diodor und Pausanias bieten kurze Erwähnungen, die auf Herodot basierend lediglich knappe zusätzliche Informationen beinhalten und nur dank des Halikarnassiers zu kontextualisieren sind ${ }^{2}$.

Dorieus' Versuch, sowohl in Libyen, als auch auf Sizilien spartanische Apoikien zu gründen, ,hat sich die Forschung nie so recht erklären können. ${ }^{\text {‘3 }}$ Dieser Beitrag wird der schmalen Quellenüberlieferung ebenfalls keine gänzliche Erklärung abgewinnen können. Aber es soll der Versuch unternommen werden, die beiden „Auswanderungsversuche“ mit der innenpolitischen Situation unter der Herrschaft des Kleomenes I., des älteren Halbbruders des Dorieus, in Verbindung zu setzen bzw. die Möglichkeit von Abhängigkeiten zu ergründen und dabei auf die herodoteische Gestaltung der Dorieus-Episode einzugehen. Dorieus wird in Teilen der Forschung als Oikist bezeichnet, der in Sparta selbst keine Aussicht auf politischen Aufstieg gehabt und deshalb aus persönlicher Motivation heraus auswärtige Betätigungsfelder gesucht habe ${ }^{4}$. Worauf gründet sich jedoch die für Sparta, ganz besonders für einen spartanischen Königssohn, untypische Motivation, in die Ferne zu ziehen - sogar wiederholt ein solches Unterfangen zu wagen?

\section{Herodots Bericht ${ }^{5}$}

Kleomenes I. und Dorieus sind, ebenso wie Leonidas und Kleombrotos, Söhne des Agiaden Anaxandridas II., der in erster Ehe mit seiner Nichte verheiratet gewesen ist. Aus dieser Verbindung ging jedoch zunächst kein Nachwuchs hervor, weshalb die Ephoren die Forderung erhoben, der König möge eine andere Ehefrau wählen. Zwar erwehrte sich Anaxandridas dieser ersten Aufforderung, machte aber nach einer weiteren von Ephoren und Geronten vorgebrachten Anweisung das Eingeständnis, eine weitere Frau zu ehelichen, was eigentlich gänzlich der spartanischen Sitte widersprach. Aus der zweiten Ehe ging schließlich Kleomenes I. als erstgeborener Sohn hervor. Aber auch die erste Ehegattin wurde nun schwanger und brachte als Sohn Dorieus zur Welt, dem bald darauf Leonidas und Kleombrotos folgten. Die zweite Ehegattin gebar dem Anaxandridas hingegen keinen weiteren Nachwuchs. Nach dem

\footnotetext{
${ }^{1}$ RE 5,2, 1905, Sp. 1558-1560.

${ }^{2}$ Diod. 4,23 u. 10,18; Paus. 3,3,9f. u. 3,16,4.

${ }^{3}$ STAUfFEnBERG, Dorieus S. 181. Die von Stauffenberg und in jüngerer Vergangenheit von BRACCESI, L'enigma S. 25ff. vorgebrachte Überlegung, Ziel der Unternehmungen des Dorieus sei das Durchbrechen der Umklammerung durch Phönizier und Perser gewesen, ist wenig glaubhaft; cf. WELWEI, Sparta S. 115 mit Anm. 205. Ebenso wenig kann der Sizilien-Zug als Anzeichen einer imperial-expansiven Politik gewertet werden; cf. THOMMEN, Lakedaimonion Politeia S. 70.

${ }^{4}$ So etwa BERVE, Gestaltende Kräfte S. 237 u. 261; KIMMERLE, Völkerrechtliche Beziehungen S. 55; Thommen, Lakedaimonion Politeia S. 69ff.; anders Clauss, Sparta S. 28f.; Fitzhardings, Spartans S. 158; HuXLEY, Early Sparta S. 77ff.

${ }^{5}$ Hdt. 5,39-48.
} 
Tod des Anaxandridas folgte ihm sein erstgeborener Sohn Kleomenes I. in der Regentschaft der Agiaden nach.

Nach seiner Schilderung der Familienkonstellation stellt Herodot die beiden

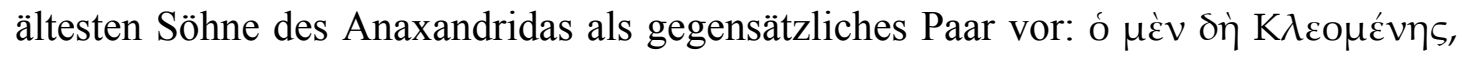

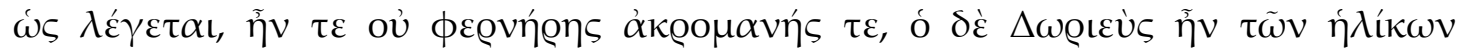

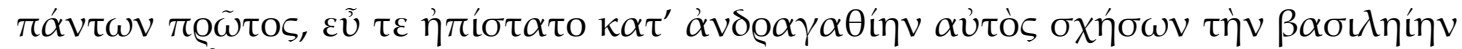
(Hdt. 5,42) ${ }^{6}$. Der nach dem Gesetz legitimierte Nachfolger ist unfähig und schwachsinnig, der zweitgeborene Sohn hingegen besitzt herausragende Qualitäten und ist sich dieser auch bewusst. Dorieus will nicht unter der Herrschaft des älteren Halbbruders leben, sondern es zieht ihn in die Ferne. Er bittet die Spartiaten um Gefolgsleute und wandert nach Libyen aus ${ }^{7}$. Schon die Angabe, Dorieus wäre bewusst gewesen, dass er besser zum Herrschen geeignet sei, könnte auf innere Streitigkeiten hinweisen. Ge-

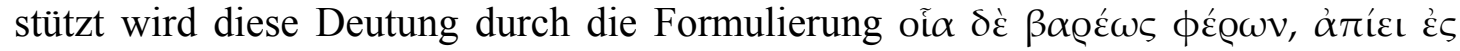
$\tau \grave{v} v \Lambda \iota \beta u ́ \eta v \tau \dot{\alpha} \pi \lambda$ oĩ $\alpha(5,42)$. Schwermütig oder wütend verlässt Dorieus Sparta. Der Versuch, in Libyen eine Apoikie zu gründen, wird nach zwei Jahren beendet, da Phönizier und ein indigener libyscher Stamm die Spartaner vertreiben. Der Sohn des Anaxandridas kehrt nach der Peloponnes zurück. Im herodoteischen Werk wird bereits durch das Ausbleiben einer Orakelbefragung in Delphi ${ }^{8}$ sowie den Verzicht auf weitere Auswanderungsriten das letztendlich unausweichliche Scheitern der LibyenExpedition angekündigt. Sollte die unterlassene Orakel-Befragung mehr sein als ein literarisches Motiv Herodots, stellt sich die Frage, warum Dorieus auf diese religiöse Praxis verzichtet hat bzw. warum man es in Sparta zuließ, dass eine Gruppe ohne Wahrung der religiös-rituellen Norm ein solches Unterfangen anging.

Auf die kurze Schilderung der Libyen-Expedition folgt bei Herodot sogleich das zweite „Auslandsunternehmen“, der Versuch, in Sizilien eine spartanische Gründung zu etablieren. Dorieus erhält von Antichares aus dem boötischen Eleon den Rat bzw. die Weissagung, Herakleia im westlichen Sizilien zu gründen ${ }^{9}$. Dies sei legitim, weil das ganze Gebiet am Fluss Eryx einst von Herakles erobert worden sei ${ }^{10}$. Den Rat lässt sich Dorieus dieses Mal durch das Delphische Orake ${ }^{11}$ bestätigen, ein besse-

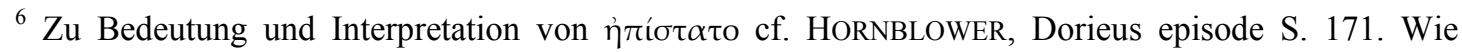
Hornblower anmerkt, ist durch die Formulierung in 5,42 die Deutung, Herodot habe die Selbsteinschätzung des Dorieus kritisch gesehen, zwar möglich, aber nicht zwingend.

${ }^{7}$ GANCI, La spedizione S. 223-231.

${ }^{8} \mathrm{Ob}$ sich der anonyme Orakelspruch Hdt. 4,178 vielleicht an Dorieus gerichtet haben könnte, wie in der Forschung vereinzelt vermutet worden ist, bleibt hypothetisch; cf. THOMMEN, Lakedaimonion Politeia S. 70 Anm. 93 mit Literatur.

${ }^{9}$ Eine eindeutige Übersetzung des herodoteischen Textes $(5,43)$ ist schwierig, da für $\kappa \tau i \zeta \zeta \iota \iota v$ sowohl die Bedeutung „gründen“, als auch ,besiedeln“ denkbar ist. Inhaltlich fällt dies jedoch nicht ins Gewicht;

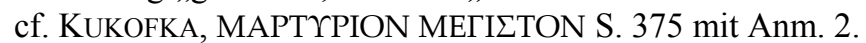

${ }^{10}$ Antichares entnahm den Rat vielleicht einer Orakelsammlung, die nach dem mythischen thebani-

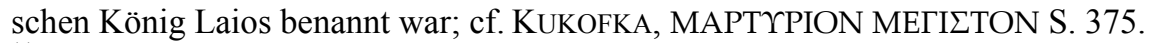

${ }^{11}$ An der Zuverlässigkeit der Information hinsichtlich des Einholens eines Orakelspruchs vor der zweiten bzw. des Verzichts auf ein Orakel vor der ersten Expedition darf man zweifeln. Vielleicht handelt es sich nur um eine aufsteigende Gliederung innerhalb des herodoteischen Berichtes. Für die Erklärung des Orakels vor der Sizilien-Expedition gibt es zwei mögliche Ansätze: Zum einen konnte sich das Orakel natürlich nicht erfüllen, da Dorieus von dem anfänglichen Vorhaben abwich und sich an der Zerstörung von Sybaris beteiligte; zum anderen könnte man auch argumentieren, dass der Orakelspruch schließlich durch Euryleons Handeln nach dem Tod des Dorieus in Erfüllung ging. Dies wird vielleicht dadurch gestützt, dass die Pythia nicht nach einem bestimmten, sondern nur allgemein nach einem Land gefragt worden ist, gegen welches Dorieus ziehen möchte. Antichares nennt ein bestimmtes Gebiet explizit, in der Orakelbefragung wird jedoch eine allgemeine Formulierung genannt $(5,43)$. $\mathrm{Zu}$

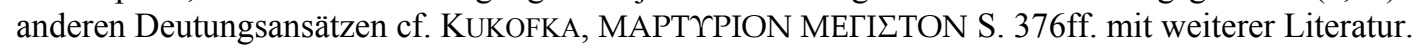


res Ende nimmt seine Unternehmung jedoch auch auf Sizilien nicht ${ }^{12}$. Der Gründungsversuch wird durch Phönizier und Egestaier vereitelt ${ }^{13}$. Dorieus findet hierbei den Tod. Für die Sizilien-Expedition nennt Herodot neben dem Anaxandridas-Sohn noch weitere spartanische $\sigma v \gamma \kappa \tau i ́ \sigma \tau \alpha \mathrm{l}^{14}$ : Thessalos, Paraibates, Keleës und Euryleon, der als einziger der namentlich bekannten überlebt und schließlich Minoa ${ }^{15}$ gründet. Dieser sammelt auch die Reste des spartanischen Heeres, was auf eine größere Gefolgschaft des Dorieus verweisen könnte. Folgt man Herodot, scheint ebenso wie die Libyen-Expedition auch der zweite Versuch auf Sizilien innerhalb des spartanischen Gemeinwesens einvernehmlich diskutiert und unternommen worden $\mathrm{zu} \operatorname{sein}^{16}$.

Herodot schließt dann die Episode mit der zum Teil kontrafaktischen Aussage, Dorieus wäre König geworden, wenn er es doch ertragen hätte, unter Kleomenes in Sparta zu verweilen. Denn der älteste Sohn des Anaxandridas hätte nicht lange gelebt und zudem keinen männlichen Nachwuchs gezeugt, sondern nur eine Tochter namens Gorgo besessen.

Die Dorieus-Expeditionen werden von Herodot nach der Schilderung des Ionischen Aufstandes (5,28-38) als analeptischer Einschub abgehandelt, dessen Anfang und Ende durch direkte Hinweise auf die Königsherrschaft des Kleomenes I. markiert werden (39,1 bzw. 49,1) $)^{17}$. Aristagoras von Milet bereist Griechenland, um für Unterstützung gegen Dareios I. zu werben, und wird auch in Sparta vorstellig. Dies bietet Herodot die Gelegenheit, nun anzumerken, dass Anaxandridas II. nicht mehr lebt, sondern Kleomenes I. jenem nachgefolgt $\mathrm{sei}^{18}$. Es folgt dann die Dorieus-Episode, die in sich geschlossen ist, im Werk von Herodot ansonsten aber keinerlei verbindende sowie inhaltlich bedeutsame Wirkung besitzt ${ }^{19}$ und für den weiteren Hergang des Berichtes, der auf die Perserkriege zuläuft, bedeutungslos bleibt. Die Episode ist inhalt-

\footnotetext{
12 ThOMmen, Lakedaimonion Politeia S. 93 erwägt, in dem Orakel-Verzicht vor der ersten Expedition ein Argument dafür zu sehen, dass Dorieus auf private Initiative hin ausgezogen sei. Jedoch verzichtete man angeblich auch auf weitere Auswanderungsriten und bemühte vor der Sizilien-Fahrt schließlich doch das Delphische Orakel.

${ }^{13}$ Schenkt man Diod. 4,23,3 Glauben, dann zerstörten die Phönizier und Egestaier die Gründung Herakleia aus Neid und Furcht, da die Siedlung schnell aufblühte.

${ }^{14}$ Paus. 3,16,4 berichtet von Anthenaiern, die mit Dorieus nach Sizilien gezogen seien. Es handelt sich hierbei um Periöken aus Kynouria, die Kleomenes vielleicht feindlich gesinnt waren; cf. THOMMEN, Lakedaimonion Politeia S. 70 Anm. 88 mit weiterer Literatur. Die Pausanias-Stelle legt nahe, dass einige der Mitstreiter des Dorieus wieder nach Sparta zurückgekehrt sind. Es muss jedoch angemerkt werden, dass die besagte Pausanias-Stelle gestört ist, der authentische Text nicht zweifelsfrei wiederhergestellt werden kann und die inhaltliche Aussage der Passage somit kaum belastbar ist. Für diesen Hinweis sei Krešimir Matijević (Trier) gedankt. Stibbe brachte einen Graffito, der auf einer in Kynouria gefunden attischen Schale angebracht ist (datiert auf ca. 525 v. Chr.) in die Diskussion ein. Zwar wird in dem kurzen Text ein Dorieus genannt, in dessen Name eine Weihung an Apollon gestiftet worden ist, die Verbindung zu dem Königssohn muss aber hypothetisch bleiben; cf. STIBBE, Das andere Sparta S. $241 \mathrm{ff}$.

${ }^{15}$ Minoa scheint in der Folge keinerlei Konflikte mit den Phöniziern gehabt zu haben; cf. Polyain. 1,28,2; StauffenberG, Dorieus S. 190. Die frühere Forschungsthese, Dorieus' Unternehmungen seien gegen eine phönizische Umklammerung gerichtet gewesen (cf. Anm. 3), ist auch unter diesem Gesichtspunkt abzulehnen. Von Euryleon berichtet Herodot weiter, er habe sich an der Befreiung Selinunts von der Tyrannis des Pheithagoras beteiligt. Das Thema Freiheit greift Herodot häufig auf, wenn er griechische Tyrannen abhandelt; cf. BROCK, Political Imagery S. 173f.

${ }^{16}$ THOMMEN, Lakedaimonion Politeia S. 70.

${ }^{17}$ HORNBLOWER, Dorieus episode S. 169.

${ }^{18}$ Erwähnung findet Kleomenes I. schon in Hdt. 3,148.

${ }^{19}$ Eine Ausnahme ist Hdt. 7,158, Gelons Werben um Hilfe für seinen Rachefeldzug. HoRNBLOWER, Dorieus episode S. 174ff. sieht in der Zerstörung von Sybaris und Milet sowie in der Darstellung des Philippus von Kroton und des Atheners Kleinias weitere markante Vergleiche, die den DorieusEinschub ,verlinken“.
} 
lich viergeteilt: Anaxandridas' Ehen und die Geburt der Söhne (5,39-41), die LibyenExpedition $(5,42)$, die Sizilien-Expedition (5,43-47) und die schließende Bemerkung über den Tod des Dorieus $(5,48)$. Mabel L. Lang hat auf die konstruierte TetradenStruktur, die der Darstellung der Auseinandersetzung zwischen Anaxandridas und den Ephoren/Geronten $\left(5,39 f\right.$.) zugrunde liegt, hingewiesen ${ }^{20}$. Es handelt sich um ein bei Herodot häufig auftretendes Darstellungsschema, das komplexe Sachverhalte für den Leser verständlich gliedert und vereinfacht.

Die Ausführungen zur Sizilien-Expedition sind am längsten, was jedoch keinerlei Wertung Herodots bedeutet, sondern lediglich daher rührt, dass der Halikarnassier hier weitere kurze „Unter-Geschichten“ einführt. Er berichtet von der Auseinandersetzung zwischen Sybaris und Kroton und bietet im Zuge einer AutopsieBehauptung die von beiden Parteien angeführten Versionen über eine mögliche Beteiligung des Dorieus auf Seiten der Krotoniaten $(5,44 \mathrm{f} \text {. })^{21}$. Da die Krotoniaten die Teilnahme Fremder an der Eroberung Sybaris' bis auf eine Ausnahme bestreiten, eröffnet sich für Herodot die Möglichkeit, auf eben diese Ausnahme, den blinden Seher Kallias von Elis, einzugehen $(5,44,2)$. Im Zuge der Schilderung der Sizilien-Expedition teilt Herodot zudem die bereits angesprochenen Namen weiterer Spartiaten mit, die an der Unternehmung beteiligt gewesen sein sollen. Da Euryleon alleinig überlebt, wird auch sein weiteres Schicksal kurz berichtet $(5,46,2)$. Schließlich kommt die Rede noch auf Philippos von Kroton, den Sohn des Butakides, der, obwohl kein Spartiate, dennoch mit Dorieus gezogen sei $(5,47)$. Die Sizilien-Expedition wird von Herodot genutzt, um ,antiquarisch“ einige kurze Informationen anzubringen, die weder für die Geschichte des Dorieus, noch für den weiteren Verlauf des herodoteischen Berichts von Relevanz sind ${ }^{22}$. Der Bericht zur Libyen-Expedition ist frei von solchen Anreicherungen, lediglich die Dorieus Hilfe leistenden Bewohner von Thera werden angemerkt. Die Vertreibung der Dorieus-Gruppe durch Phönizier und die indigenen, wohl nomadischen Maken $(5,42)$ passt in das bei Herodot mehrmals greifbare Schema, nach welchem die militärisch tüchtigen und zudem stets mobilen Nomaden überlegene und gut organisierte Heere besiegen ${ }^{23}$.

Wie erklärt sich die Position der Dorieus-Episode unmittelbar nach dem Bericht, dass nun, als Aristagoras von Milet sich hilfesuchend an Sparta wendet, Kleomenes I. auf den verstorbenen Anaxandridas gefolgt sei? Vielleicht kann die Anordnung der Episode so verstanden werden, dass Herodot dem Leser erklären will, wie der neue Agiaden-König zur Herrschaft gelangt ist. Die einfache Anmerkung, dass er der älteste Sohn und legitime Nachfolger gewesen ist, wäre ausreichend und würde nicht von der „Haupthandlung“ wegführen, verbietet sich jedoch ob der besonderen Umstände, der Familienkonstellation und der daraus erwachsenen Spannungen ${ }^{24}$.

Herodot wiederholt in gewisser Hinsicht mit der Dorieus-Episode die im vierten Buch $^{25}$ gebotene Erzählung von Theras ${ }^{26}$, der für seine unmündigen Neffen zu-

\footnotetext{
${ }^{20}$ LANG, Herodotean Narrative S. 106ff. mit weiteren Stellen.

${ }^{21}$ Die im herodoteischen Bericht eingesetzte Zwischenstation des Dorieus in Italien erinnert an die Unternehmungen des Spartiaten Gylippus aus dem Jahr 413 v. Chr. Dieser machte auf dem Weg nach Sizilien in Libyen Halt und entsetzte das von Libyern belagerte Euesperia; cf. Thuk. 7,50.

${ }^{22}$ Die Erwähnung der Egestaier in der Dorieus-Episode ist eher inhaltsleer, was der allgemeinen herodoteischen Darstellung der Bewohner von Sardinien, Korsika und Sizilien entspricht; cf. BICHLER, Herodots Welt S. 75.

${ }^{23}$ BiCHLER, Herodots Welt S. 100; Bichler/Rollinger, Herodot S. 53.

${ }^{24}$ Ähnliche Deutung bei HornBlOWER, Dorieus episode S. 170.

${ }^{25}$ Hdt. 4,146-149; cf. BARAgWANATH, Motivation S. 165ff. Die Theras-Episode ist Teil des Berichts über die Libyen-Kampagne des Satrapen Aryandes. Auch hier findet sich also eine der DorieusEpisode gemeinsame Verbindung von Thera und Libyen.
} 
nächst das Königsamt in Sparta verwaltet, dann später jedoch nicht mehr unter deren Herrschaft leben will. Er fasste den Plan, eine auswärtige Kolonie zu gründen. Dafür bittet er um die in Sparta zwischenzeitlich inhaftierten Minyer, die nach der Beteiligung an der spartanischen Königsherrschaft gestrebt haben. Die Minyer wurden von ihren listigen Frauen aus der Haft befreit, entkamen schließlich getarnt durch weibliche Kleidung und verschanzten sich auf dem Taygetos. Theras bittet in Sparta nun erfolgreich darum, die Minyer aus dem Gemeinwesen fortführen zu dürfen. Die Motivation für das Verlassen des spartanischen Gemeinwesens ist also in doppelter Hinsicht die Nicht-Akzeptanz der legitimen Herrschaft.

Ein historischer Kern der Theras-Erzählung ist nicht fassbar, vielmehr handelt es sich um eine fiktive Geschichte. Da durch Theras schließlich Thera gegründet wird, liegt wohl eine aitiologische, retrospektiv entwickelte Erzählung für die Gründung der Stadt vor ${ }^{27}$.

\section{Ein kritischer Deutungsversuch}

Der herodoteische Bericht über die beiden Expeditionen zeigt zu Beginn der kurzen Schilderung der Libyen-Unternehmung implizit auf, dass es zwischen den ungleichen Halbbrüdern zu Zwistigkeiten gekommen sein könnte. Dorieus sieht sich selbst, verglichen mit Kleomenes I., als besser geeigneten König an, verlässt dann jedoch Sparta im Zorn, ohne die üblichen religiösen Gepflogenheiten zu beachten. Dass er nach Gefolgsmännern fragt, die ihm auch mitgegeben werden, suggeriert hingegen ein einvernehmliches Unternehmen, das auf allgemeinen Konsens im spartanischen Gemeinwesen gestoßen ist. Hier scheint ein Widerspruch greifbar. Einem machtbewussten und ehrgeizigen Königsohn, der in die Ferne ziehen möchte, werden spartanische Vollbürger anvertraut, aber auf die üblichen Riten wird verzichtet? Vermutlich herrschte zwischen Kleomenes I. und Dorieus von Beginn an ein angespanntes Verhältnis, das sich nach dem Tod des gemeinsamen Vaters gänzlich zu einem Bruch steigerte, von dem wohl auch Teile der Aristokratie betroffen waren. Bereits während der Schwangerschaft gingen die Angehörigen der zweiten Ehefrau gegen die Mutter des Dorieus vor und unterstellten ihr, sie würde dem Anaxandridas ein Kind unterschieben ${ }^{28}$. Im herodoteischen Bericht kontrollieren die Ephoren die Niederkunft der ersten Ehefrau. Die Verwandten der Mutter des Kleomenes I. haben aus eigenem Interesse die Rechtmäßigkeit der Abkunft des Dorieus von Anaxandridas bestritten, was die Präsenz der Ephoren bei der Geburt auf den Plan rief ${ }^{29}$. Unweigerlich fühlt man sich an die spätere Absetzung des Eurypontiden Damaratos erinnert, die durch Kleomenes I. betrieben worden ist und weiter unten noch zu thematisieren sein wird.

Offensichtlich gab es bereits vor der Geburt des Dorieus zwischen den Familien der beiden Ehefrauen offenen Streit ob der Nachfolgefrage. Dass auf Dorieus schließlich mit Leonidas und Kleombrotos noch zwei weitere Söhne aus der Ehe des Anaxandridas mit seiner Nichte, der ersten Frau, folgten, dürfte die Streitfrage zusätzlich angeheizt haben. Es ist auffällig, dass Anaxandridas bei Herodot keine Rolle

\footnotetext{
${ }^{26}$ Theras selber war kein gebürtiger Spartaner, sondern stammte aus Boötien; cf. MAVROGIANNIS, Herodotus S. 60.

${ }^{27}$ THOMMEN, Sparta S. 29.

${ }^{28}$ Hdt. 5,41,2. Platon berichtet, die Ephoren hätten grundsätzlich die Königsgattinnen überwacht (Alk. 1,121), was jedoch zurückzuweisen ist; cf. LinK, Kosmos Sparta S. 125 Anm. 6.

${ }^{29}$ Zur Aufsichtsfunktion der Ephoren bei Nachfolgeregelungen sowie zum Zusammenspiel von Ephorat und Gerusia: ThOMMEn, Lakedaimonion Politeia S. 78ff.; LuTHER, Könige und Ephoren. S. 94ff.; SOMMER, Ephorat S. 39ff.
} 
spielt. Als König hatte er sich zunächst der Anweisung der Ephoren, seine erste Frau

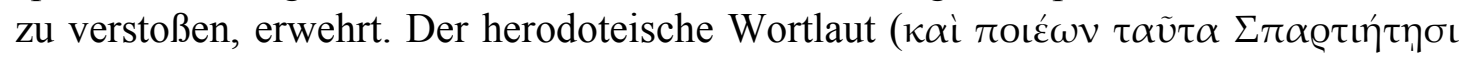

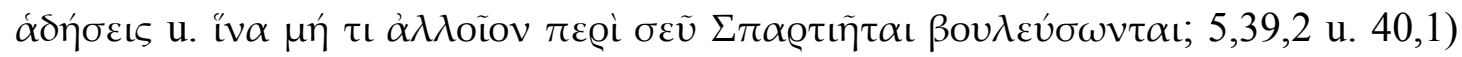
könnte ferner eine allgemeine Missstimmung gegenüber Anaxandridas anzeigen. Als sich der König zunächst widersetzte, ist es eine von Ephoren und Geronten gemeinsam vorgetragene Anweisung, der er sich schließlich fügt. Anaxandridas erscheint als ein streitbarer Agiade, der eine enge Bindung an seine erste Frau hatte ${ }^{30}$. Dieser Faktor dürfte sich bei dem Streit um die Nachfolge ebenfalls kaum schlichtend ausgewirkt haben. Vielmehr dürfte sich Anaxandridas nach der Geburt der drei Söhne Dorieus, Leonidas und Kleombrotos in dem ursprünglichen Widerstreben, seine Ehefrau zu verstoßen und eine neue Frau zu ehelichen, mehr denn je bestärkt gefühlt haben. Es drängt sich ferner die Frage auf, warum die Familie der Kleomenes-Mutter gegen die erste Ehefrau vorging, wenn doch ohnehin - wie dann auch erfolgt - der älteste Sohn die Nachfolge im Agiaden-Haus antreten sollte? ${ }^{31}$

Von Interesse ist in dieser Hinsicht vielleicht auch der Name des zweitgeborenen Sohnes: „der Dorier“. In der Forschung wurde darauf hingewiesen, dass der Name eventuell einen programmatischen Impetus besessen haben könnte ${ }^{32}$. In spätarchaischer Zeit lassen sich Belege dafür finden, dass man in Sparta bewusst an die achaische Vergangenheit der Peloponnes anknüpfen wollte ${ }^{33}$, in der die Anfänge des Peloponnesischen Bundes liegen. Kleomenes selbst soll in Athen aufgetreten sein und sich

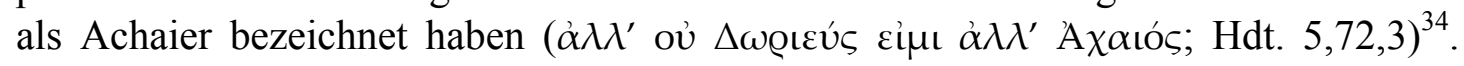
Dieser Ausspruch ist freilich mehr als kritisch zu sehen und muss als fiktiv verworfen werden. Vielleicht ist hier eine allgemeine spartanische Ausrichtung auf das achaische Erbe greifbar, die im herodoteischen Werk verdichtet in einem Ausspruch des Königs dargelegt wird. Was bezweckten aber Anaxandridas und seine Frau mit dem Namen „Dorieus“, der explizit an die dorischen Wurzeln der Spartaner erinnerte, der bewusst umgesetzten Anlehnung an die mythische Frühzeit der Peloponnes allerdings entgegenstand?

Leonidas und Kleombrotos, die Brüder des Dorieus, sowie dessen leiblicher Sohn Euryanax ${ }^{35}$ zogen nicht mit nach Libyen und Sizilien. Sie verblieben in Sparta, was der hier dargelegten These eines Streites innerhalb der Agiaden-Familie auf den ersten Blick zu widersprechen scheint. Vielleicht waren Leonidas und Kleombrotos aufgrund ihres jüngeren Alters keine Bedrohung für Kleomenes. Da der älteste Sohn stets die Nachfolge antrat, bestand das Streitpotenzial wohl vielmehr in der Frage nach der Gültigkeit und dem legitimen Anspruch der zweiten Ehe bzw. der Abstammung des Kleomenes aus eben dieser Ehe. Für Kleomenes war folglich in erster Linie Dorieus eine Bedrohung ${ }^{36}$.

Die Regentschaft des Kleomenes I. ${ }^{37}$ war gekennzeichnet von innenpolitischen Krisenmomenten, die aus seinem außenpolitischen Handeln resultierten. Nach der

\footnotetext{
${ }^{30}$ In der Forschung wurde als Erklärung für die enge Bindung an die erste Ehefrau deren Vermögenstand in Erwägung gezogen; cf. HodKInSON, Land Tenure S. 400; LINK, Das frühe Sparta. S. 2.

${ }^{31}$ In der Darstellung des Pausanias $(3,3,10)$ geben die spartanischen Vollbürger nur widerwillig dem legitimen Nachfolger den Vorrang vor Dorieus.

${ }^{32}$ CARTLEDGE, Spartans S. 93; HuXLEY, Early Sparta S. 71.

${ }^{33}$ STE. Croix, Sparta’s 'Foreign Policy' S. 219; ThOMmen, Sparta S. $51 \mathrm{f}$.

${ }^{34}$ HornBLOWER, Dorieus episode S. 169.

${ }^{35}$ Hdt. 9, 10 .

${ }^{36}$ Da Kleomenes I. keinen männlichen Nachwuchs hatte, könnte die Anwesenheit potentieller Nachfolger aus dem Haus der Agiaden vielleicht sogar durch die Ephoren erzwungen worden sein.

${ }^{37} \mathrm{Zu}$ der hier nur kurz referierten Geschichte des Kleomenes I.: THOMMEN, Sparta S. 63ff.; WeLWEI, Sparta S. 106ff.; WeLWEI, Kleomenes I. und Pausanias S. 37-45.
} 
erfolgreichen Intervention in Athen 510 v. Chr. ${ }^{38}$ scheiterte 508/7 v. Chr. Kleomenes' Plan, Isagoras anstelle von Kleisthenes zu installieren. Der in unmittelbarer Folge unternommene Zug im Jahr 507/6 v. Chr. blieb erfolglos, da sich der zweite spartanische König Damaratos sowie Teile der Bundesgenossen gegen Kleomenes I. stellten. Damaratos konnte in Sparta vermutlich auf entsprechenden Rückhalt vertrauen und es somit wagen, sich mit Kleomenes I. zu überwerfen ${ }^{39}$. Wie intensiv der Streit der beiden Könige gewesen ist, wird durch institutionelle Veränderungen ersichtlich, die als Folge des Jahres 507/6 v. Chr. umgesetzt worden sind. Zukünftig sollten die Könige nicht mehr gemeinsam ein Heer führen dürfen ${ }^{40}$. Die nächste innere Krise ergab sich für Kleomenes 494 v. Chr., als er aufgrund des unterlassenen Angriffs auf Argos in Sparta angeklagt wurde. Das Urteil wurde von der Apella gesprochen und hatte einen Freispruch zur Folge, woraus vielleicht der Schluss gezogen werden darf, dass der Widerstand gegen den König nicht allgemein von den Vollbürgern, sondern eher von Kreisen der Aristokratie ausging. Ferner soll Damaratos gegen den Agiaden intrigiert haben, als dieser versuchte, einen befürchteten Medismos in Aigina zu unterbinden ${ }^{41}$. Kleomenes I. ging nun entschieden gegen Damaratos vor und fand in dem Eurypontiden Leotychidas, der mit Damaratos verfeindet gewesen sein soll, einen willkommenen Helfer. Leotychidas soll geschworen haben, dass Damaratos nicht der leibliche Sohn des Ariston $\mathrm{se}^{42}$. Zeugenaussagen und schließlich ein Delphisches Orakel, das Kleomenes I. durch Bestechung in seinem Sinne beeinflusst haben soll, führten zur Absetzung des Damaratos ${ }^{43}$. Die Bestechung des Delphischen Orakels, die später offengelegt worden ist, wurde Kleomenes I. schließlich zum Verhängnis und führte zu seinem Exil in Arkadien.

Die gegen Kleomenes I. auftretende Opposition - Herodot spricht wörtlich

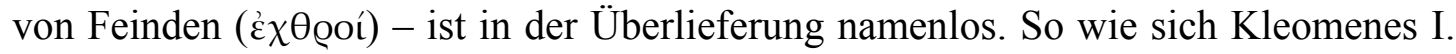
an dem Damaratos feindlich gesinnte Angehörige des Eurypontiden-Hauses wandte, dürfte auch der Kreis um Damaratos im Agiaden-Haus nach Gegnern des Kleomenes I. gesucht haben. $\mathrm{Zu}$ denken ist in erster Linie an Leonidas, Kleombrotos und Euryanax, ferner darf auch an die innerfamiliären Streitigkeiten vor der Geburt des Dorieus erinnert werden. In der Forschung wurde bereits häufig diskutiert, ob Kleomenes I. letztlich vielleicht ermordet worden ist. Herodot erzählt von dem Vorwurf, der aus dem Exil nach Sparta zurückgerufene König sei geisteskrank geworden, habe sich verstümmelt und schließlich selbst dem Tod zugeführt. Entschleiert man den herodoteischen Bericht, wird die Nachricht über den angeblichen Wahnsinn und Selbstmord des Kleomenes als mögliche Vertuschung eines Mordkomplotts deutbar. Der Vorwurf des Wahnsinns kann womöglich als eine bewusst von der KleomenesOpposition verbreitete Geschichte angesehen werden ${ }^{44}$. 'ExӨoó, sowohl unter den Eurypontiden, als auch unter der eigenen Agiaden-Familie, hatte Kleomenes I. sicherlich genug ${ }^{45}$.

\footnotetext{
${ }^{38}$ Da sich die Entscheidung, in Athen einzugreifen, verzögerte, könnte schon damals eine starke Opposition gegen Kleomenes am Werk gewesen sein; cf. WELWEI, Kleomenes I. und Pausanais S. 39.

${ }^{39}$ WeLWEI, Kleomens I. und Pausanais S. 40.

${ }^{40}$ Hdt. 5,74f.; LUTHER, Ephoren und Könige S. 105ff.

${ }^{41}$ Hdt. 6,61-65,4.

${ }^{42}$ Zur Geburt des Damaratos: Hdt. 6,63ff.; LuTHER, Ephoren und Könige S. 99ff.

${ }^{43}$ Diese religiöse Freveltat markiert in der herodoteischen Darstellung einen Wendepunkt in der Geschichte des Königs. Die Verbindung von Bestechung bzw. Betrug und einem unwürdigen Ende ist bei Herodot als Darstellungsmotiv verbreitet; cf. BICHLER, Historiographie S. 20f. Zur Pietätlosigkeit des Kleomenes bzw. zu deren Darstellung bei Herodot auch IMMERWAHR, Form and Thought S. 192f.

${ }^{44}$ Cf. Anm. 37 u. 43.

${ }^{45}$ HARVEY, Leonidas S. 253-260.
} 
Betrachtet man den innenpolitischen Gegenwind, mit dem Kleomenes während seiner Regentschaft zu kämpfen hatte, wäre es von großem Interesse zu wissen, wann genau Dorieus von seiner ersten Expedition zurückkehrte. Sein neuerliches Erscheinen dürfte für Kleomenes I. sicherlich nicht positiv, der aristokratischen Opposition hingegen sehr zuträglich gewesen sein. Hinsichtlich der Datierung der beiden Expeditionen gibt es jedoch kaum sichere Ansätze ${ }^{46}$. Selbst der Tod des Anaxandridas ist nicht gesichert, wird jedoch meist in die Zeit um 520 v. Chr. datiert. Obwohl der herodoteische Bericht dies suggeriert, muss man nicht zwingend annehmen, dass Dorieus Sparta in Richtung Libyen verlassen hat, unmittelbar nachdem Kleomenes die Nachfolge angetreten hatte; ebenso wenig ist davon auszugehen, dass nach der Libyen- sogleich die Sizilien-Expedition erfolgte. In Libyen verweilte Dorieus mit seinen Anhängern nach Herodot zwei Jahre. Einen chronologischen Ansatzpunkt bietet Euryanax, der Sohn des Dorieus. Dieser war 479 v. Chr., als er Pausanias zur Schlacht von Platää begleitete, wohl im besten Mannesalter. Vielleicht darf man seine Zeugung in die Zeit zwischen 515-500 v. Chr. datieren und somit die phasenweise Anwesenheit des Dorieus in Sparta innerhalb dieses Zeitfensters behaupten, gleichwohl dies hypothetisch bleiben muss. Dass Euryanax seinen Vater nicht nach Sizilien begleitet hat, könnte durch sein junges Alter zu erklären sein. Politisch dürfte Euryanax in Sparta keinerlei Ambition gehabt haben, denn obwohl Kleomenes I. keinen männlichen Nachkommen zeugte, waren mit Leonidas und Kleombrotos ältere männliche Angehörige, die zudem Königssöhne waren, im Haus der Agiaden vorhanden. Für Euryanax wäre somit als Sohn des Dorieus in einer von diesem gegründeten Apoikie eine deutlich komfortablere Situation möglich gewesen ${ }^{47}$.

Welche Zeitspanne zwischen der Libyen- und der Sizilien-Expedition lag, ist nicht zu sagen. Laut Herodot besteht zwar die Möglichkeit, dass sich Dorieus an der Zerstörung von Sybaris (ca. 510 v. Chr.) beteiligt haben könnte, jedoch wurde dies von der Forschung zurückgewiesen ${ }^{48}$. Als gesicherte Termini ante quem können lediglich der Antritt der Königsherrschaft durch Leonidas ca. 490 v. Chr. sowie der Rachekrieg des Gelon aus dem Jahr ca. 489 v. Chr. angeführt werden. Gelon betonte in Sparta die Erinnerung an Dorieus und versuchte, auf diese Weise spartanische Hilfe zu gewinnen ${ }^{49}$. Die Gelon-Notiz bei Herodot, sofern es sich nicht um eine Erfindung des Halikarnassiers handelt, zeigt eine positiv konnotierte Erinnerung an Dorieus in Sparta, freilich in einer Zeit nach Kleomenes I. ${ }^{50}$

Abschließend muss noch angemerkt werden, dass die Gründung von Apokien für Sparta untypisch gewesen ist. Die einzige spartanische Kolonie ist Tarent, das von den sog. Partheniai gegründet worden sein soll ${ }^{51}$. Die „Jungfrauenkinder“ stammten zwar von Vollbürgern ab, jedoch ist ihr Auszug aus Sparta in Verbindung mit inneren

\footnotetext{
${ }^{46}$ Ausführlich zur Chronologie der Dorieus-Episode: MERANTE, cronologia S. 272-294.

${ }^{47}$ Eine erfolgreiche Koloniegründung wäre politisch vermutlich als Tyrannis organisiert worden; cf. Munson, An alternate world S. 261f.

${ }^{48}$ Cf. Anm. 1; vorsichtiger WELWEI, DNP 3, 1997, Sp. 778f.

${ }^{49}$ Hdt. 7,158; STAUFFENBERG, Dorieus S. 190ff.

${ }^{50}$ Die Einschätzung von KIMMERLE, Völkerrechtliche Beziehungen S. 55f. mit Anm. 210, es habe sich bei Dorieus' Sizilien-Expedition um eine rein private Unternehmung gehandelt, da man ansonsten in Sparta nicht auf Gelons Hilfegesuch ablehnend reagiert hätte, ist zurückzuweisen. Zu dieser Zeit konnte sich Sparta, im Anbetracht der drohenden Persergefahr, schwerlich auf Unternehmungen in Sizilien einlassen. Somit kann über die Umstände der Dorieus-Unternehmung keine Einschätzung abgeleitet werden. Interessant ist die von HORNBLOWER, Dorieus episode S. 183 aufgeworfene, aber nicht untersuchte Frage, warum Kleomenes I. nicht zeitnah an „Rache“ für Dorieus gedacht habe. Eine schlüssige Antwort ergibt sich eo ipso aus der in diesem Beitrag vorgetragenen These.

${ }^{51}$ MeIER, Aristokraten und Damoden S. 121ff.; ThOmmen, Sparta S. 29f.; MeIER, Homoios-Ideal S. $120 f$.
} 
sozialen Unruhen zu sehen ${ }^{52}$. Der für spartanische Verhältnisse außergewöhnliche Schritt, eine Kolonie in der Fremde zu gründen, ist vor dem hier skizzierten Hintergrund auch im Falle von Dorieus durch innere Streitigkeiten zu erklären. Verbindet man die beiden Dorieus-Expeditionen mit der innenpolitisch turbulenten Zeit der Regentschaft Kleomenes' I. liegt diese Deutung nahe: Wie die Gründung Tarents, so waren auch die Versuche, in Libyen und Sizilien Apokien zu errichten, Symptome innerer Unruhen in Sparta. Der Auszug des Dorieus muss als Resultat dieser Streitigkeiten angesehen werden, ist zugleich aber auch als ein den inneren Zwist schlichtender Vorgang zu werten. Hier die Frage anzuschließen, wer oder was genau Dorieus zum Verlassen des lakedaimonischen Gemeinwesens wiederholt motivierte oder gezwungen hat, ist freilich naheliegend, gleichwohl muss eine Antwort ausbleiben.

Herodots Schweigen hinsichtlich eines intensiven Streites im Agiaden-Haus könnte darauf zurückzuführen sein, dass er hier eine offizielle, von der Partei um Kleomenes I. verbreitete Version der Ereignisse verarbeitet hat, in welcher ein Konflikt innerhalb der Agiaden-Familie natürlich nicht zur Sprache kam. Selbst in der Zeit der Regentschaft des Leonidas (ca. 490-480 v. Chr.) dürfte man zwar positiv an Dorieus erinnert, aber die innerfamiliären Zwistigkeiten vergangener Tage aus der Memorierung getilgt haben ${ }^{53}$. Ebenso griff Herodot auch eine dem Kleomenes feindlich gesinnte Darstellung auf, die den König als wahnsinnigen Irren umschrieb ${ }^{54}$. Diese konträren Nachrichten trug Herodot unkritisch zusammen, vermischte sie in seiner Darstellung und nutzte den Dorieus-Einschub lediglich, um Einzelinformationen, die keinen weiteren Bezugspunkt hatten, in sein Werk integrieren zu können. Die Ausführung zu Dorieus ist weder für das ihn umgebende Thema (Aristagoras von Milet bittet um Hilfe), noch für den weiteren Aufbau und Hergang des Berichts über die Perserkrieg relevant und zudem von typischen ,herodoteischen“ Erzählmotiven durchsetzt.

Die Gemeinsamkeiten und Übereinstimmungen zwischen der einer frühen mythischen Zeit entstammenden, aber nicht historischen Theras-Erzählung und der Dorieus-Episode wurden bereits angesprochen, eine gegenseitige Beeinflussung ist kaum von der Hand zu weisen ${ }^{55}$ : In beiden Fällen geht es um eine Beteiligung von nicht legitimierten Personen an der politischen Herrschaft und einer aus dieser Span-

\footnotetext{
${ }^{52}$ Nach Aristoteles wurden sie bei einer Verschwörung ertappt (pol. 1306b 30f.). Zum Ursprung der Partheniai liegen zwei unterschiedliche Überlieferungstraditionen vor. Nach Antiochos von Syrakus waren sie Nachkommen von Spartiaten, die nicht am ersten Messenischen Krieg teilgenommen hatten. Deren Nachfahren seien deshalb als nicht vollberechtigt anerkannt worden. Ephoros hingegen berichtet, im zehnten Jahr des ersten Messenischen Krieges seien die jüngsten und stärksten Spartiaten aus Messenien nach Sparta geschickt worden, um dort Nachfahren zu zeugen. Diese seien jedoch später als in ihrem Rechtsstatus verminderte Bürger angesehen und bei der Verteilung von Landlosen benachteiligt worden. Der historische Kern der beiden Überlieferungstraditionen, die von Strabon $(6,3,2 \mathrm{f}$.) bewahrt worden sind, ist nicht zu ergründen. Sicher ist jedoch, dass der Auszug einer spartanischen Gruppierung und die daraus resultierende Gründung Tarents mit sozialen Unruhen in Sparta einhergingen.

${ }^{53}$ Leonidas ehelichte Gorgo, die einzige Tochter des Kleomenes I., wodurch die beiden Familienzweige der Agiaden vereint und die Streitigkeiten wohl beigelegt wurden: Hdt. 7,205 u. 239. Genauso wie sein Vater Anaxandridas heiratete Leonidas seine Nichte.

${ }^{54}$ Hdt. 5,42; 6,75; WeLWEI, Kleomens I. und Pausanais S. 45; cf. auch Anm. 37 u. 43. Allerdings könnte es sich auch um ein bloßes Darstellungsmotiv handeln. Herodot gebraucht das Motiv des Königs, der gegen Ende seines Lebens wahnsinnig wird, auch bei seiner Darstellung des Kambyses; cf. BICHLER, Historiographie S. 37f.

${ }^{55}$ Die Nennung von Thera im Zuge der Erläuterung der Libyen-Expedition eröffnet die Möglichkeit einer assoziativen Erinnerung an die im vorigen Buch berichtete Theras-Geschichte. Die gleiche inhaltliche Thematik beider Episoden könnte somit von Herodot bewusst gesetzt und vom Leser gezielt wahrgenommen worden sein. Zu einer weiteren, letztlich im Mythos wurzelnden Interpretation der Thera-Erwähnung cf. STAUfFENBERG, Dorieus S. 198ff.
} 
nungssituation resultierenden Auswanderung. Die Theras-Erzählung führt vor Augen, was in der Dorieus-Episode lediglich in Zwistigkeiten zwischen den Angehörigen der beiden Ehefrauen angedeutet wird: blutige Auseinandersetzungen, erbitterter Streit um die Königsherrschaft und eine innere Spaltung des Gemeinwesens. Spiegelt Herodot in seinen Ausführungen zu Theras die nicht explizit genannten, aber mehr als wahrscheinlichen inneren Machtkämpfe zwischen dem angeblich, am besten zur Herrschaft geeigneten“ Dorieus und dem ,wahnsinnigen“ Kleomenes I.? 


\section{Literatur}

BARAGWANATH, Motivation

E. BARAgWANATH, Motivation and Narrative in Herodotus, Oxford 2008.

BERVE, Gestaltende Kräfte

H. BERVE, Gestaltende Kräfte der Antike. Aufsätze und Vorträge zur griechischen und römischen Geschichte, München 1966.

BICHLER, Herodots Welt

R. BICHLER, Herodots Welt. Der Aufbau der Historie am Bild der fremden Länder und Völker, ihrer Zivilisation und ihrer Geschichte, ${ }^{2}$ Berlin 2001.

BICHLER, Historiographie

R. BICHLER, Historiographie - Ethnographie - Utopie. Gesammelte Schriften, Teil 1. Studien zu Herodots Kunst der Historie, hrsg. v. R. Rollinger, Wiesbaden 2007.

BICHLER/ROLLINGER, Herodot

R. BICHLER/R. RolLINGER, Herodot, ${ }^{3}$ Hildesheim u.a. 2011.

BRACCESI, L'enigma

L. BRACCESI, L'enigma Dorieo, Rom 1999.

BROCK, Political Imagery

R. BROCK, Political Imagery in Herodotus, in: V. KARAGEORGHIS / I. TAIFACOS

(Ed.), The World of Herodotus, Nicosia 2004, S. 169-177.

CARTLEDGE, Spartans

P. CARTLEdge, The Spartans. The World of the Warrior-Heroes of Ancient Greece, New York 2003.

Clauss, Sparta

M. Clauss, Sparta. Eine Einführung in seine Geschichte und Zivilisation, München 1983.

FITZHARDINGS, Spartans

L.F. FITZHARDINGS, The Spartans, London 1980.

GANCI, La spedizione

R. GANCI, La spedizione di Dorieo in Libia, in: Hesperia 5 (1995) S. 223-231.

HARVEY, Leonidas

D. HARVEY, Leonidas the Regicide? Speculations on the Death of Kleomenes I, in: G.W. Bowersock U.A. (Ed.), Arktouros. Hellenic Studies presented to B.M.W. Knox on the Occasion of his 65th Birthday, Berlin/New York 1979, S. 253-260.

HoDKINSON, Land Tenure

S. HodkInson, Land Tenure and Inheritance in Classical Sparta, in: CQ 36 (1986) S. 378-406.

HORNBLOWER, Dorieus episode

S. Hornblower, The Dorieus episode and the Ionian Revolt $(5,42-8)$, in: E. IRWIN /E GREENWOOD (Ed.), Reading Herodotus. A Study of the Logoi in Book 5 of Herodotus' Histories, Cambridge 2007, S. 168-178. 
HUXYLEY, Early Sparta

G.L. HuXYLEY, Early Sparta, London 1962.

IMMERWAHR, Form and Thought

H.R. IMMERWAHR, Form and Thought in Herodotus, Cleveland 1966.

KIMMERLE, Völkerrechtliche Beziehungen

R. KIMMERLE, Völkerrechtliche Beziehungen Spartas in spätarchaischer und frühklassischer Zeit, Münster 2005.

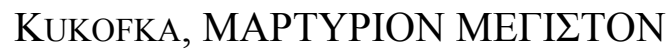

D.-A. KUKOFKA, MAPTYPION MEГI $\Sigma T O N$ der Sybariten (Herodot 5,43-46), in: Hermes 119 (1991) S. 374-380.

LANG, Herodotean Narrative

M.L. LANG, Herodotean Narrative and Discourse, Cambridge/London 1984.

LINK, Kosmos Sparta

ST. LinK, Der Kosmos Sparta. Recht und Sitte in klassischer Zeit, Darmstadt 1994.

LINK, Das frühe Sparta

ST. LINK, Das frühe Sparta. Untersuchungen zur spartanischen Staatsbildung im 7. und 6. Jahrhundert v. Chr., St. Katharinen 2000.

LUTHER, Könige und Ephoren

A. LUTHER, Könige und Ephoren. Untersuchungen zur spartanischen Verfassungsgeschichte, Frankfurt a.M. 2004.

MAVROGIANNIS, Herodotus

T. MAVRogiannis, Herodotus and the Phoenicians, in: V. KarageOrghis / I.

TAIfACOS (Ed.), The World of Herodotus, Nicosia 2004, S. 53-71.

MEIER, Aristokraten und Damoden

M. MEIER, Aristokraten und Damoden. Untersuchungen zur inneren Entwicklung Spartas im 7. Jahrhundert v. Chr. und zur politischen Funktion der Dichtung des Tyrtaios, Stuttgart 1998.

MEIER, Homoios-Ideal

M. MeIER, Wann entstand das Homoios-Ideal in Sparta?, in: A. LUTHER/M.

Meier/L. Thommen (Hrsg.), Das frühe Sparta, Stuttgart 2006, S. 113-124.

MERANTE, cronologia

V. Merante, Sulla cronologia di Dorieo e su alcuni problemi connessi, in: Historia 19 (1970) S. 272-294.

Munson, An alternate world

R.V. Munson, An alternate world: Herodotus and Italy, in: C. Dewald/J. Marincola (Ed.), The Cambridge Companion to Herodotus, Cambridge 2006, S. 257-273.

SOMMER, Ephorat

ST. Sommer, Das Ephorat: Garant des spartanischen Kosmos, St. Katharinen 2001. 


\section{STAUFFENBERG, Dorieus}

A. Schenk Graf von Stauffenberg, Dorieus, in: Historia 9 (1960) S. 181215.

STE. CRoIX, Sparta's 'Foreign Policy'

G.E.M. DE STE. CroiX, Sparta's 'Foreign Policy', in: M. Whitby (Ed.), Sparta, New York 2002, S. 218-222.

STIBBE, Das andere Sparta

C. STIBBE, Das andere Sparta, Mainz 1996.

THOMMEN, Lakedaimonion Politeia

L. Thommen, Lakedaimonion Politeia. Die Entstehung der spartanischen Verfassung, Stuttgart 1996.

THOMMEN, Sparta

L. ThOMmen, Sparta. Verfassungs- und Sozialgeschichte einer griechischen Polis, Stuttgart/Weimar 2003.

WELWEI, Sparta

K.-W. WelweI, Sparta, Aufstieg und Niedergang einer antiken Großmacht, Stuttgart 2004.

WelweI, Kleomenes I. und Pausanias

K.-W. WelWeI, Kleomenes I. und Pausanias: Zum Problem von Einzelpersönlichkeiten und Polis in Sparta im späten 6. und frühen 5. Jahrhundert v. Chr., in: B. BleCKMAnN (Hrsg.), Herodot und die Epoche der Perserkriege. Realitäten und Fiktionen. Kolloquium zum 80. Geburtstag von Dietmar Kienast, Köln u.a. 2007, S. 37-52.

\section{Kontakt zum Autor:}

Patrick Reinard, M.A.

Seminar für Alte Geschichte

Philipps-Universität Marburg

Wilhelm-Röpke-Str. 6C

35032 Marburg

reinard@staff.uni-marburg.de 